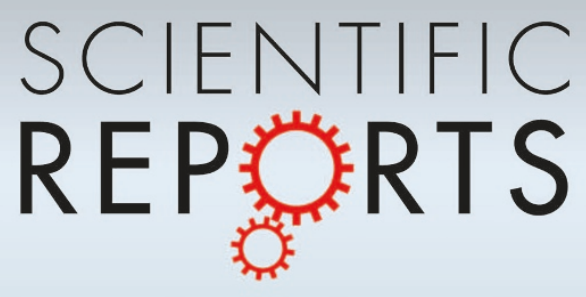

OPEN All Carbon-Based Photodetectors: An

SUBJECT AREAS:

MATERIALS SCIENCE

MATERIALS FOR DEVICES

NANOSCALE MATERIALS

ELECTRONIC PROPERTIES AND

DEVICES

\title{
eminent integration of graphite quantum dots and two dimensional graphene
}

\author{
Shih-Hao Cheng, Tong-Min Weng, Meng-Lin Lu, Wei-Chun Tan, Ju-Ying Chen \& Yang-Fang Chen
}

Department of Physics, National Taiwan University, Taipei 106, Taiwan.

Received

8 April 2013

Accepted

30 August 2013

Published

18 September 2013

Correspondence and requests for materials should be addressed to Y.F.C. (yfchen@phys. ntu.edu.tw)
Photodetectors with ultrahigh sensitivity based on the composite made with all carbon-based materials consisting of graphite quantum dots (QDs), and two dimensional graphene crystal have been demonstrated. Under light illumination, remarkably, a photocurrent responsivity up to $4 \times 10^{7} \mathrm{AW}^{-1}$ can be obtained. The underlying mechanism is attributed to the spatial separation of photogenerated electrons and holes due to the charge transfer caused by the appropriate band alignment across the interface between graphite QDs and graphene. Besides, the large absorptivity of graphite QDs and the excellent conductivity of the graphene sheet also play significant roles. Our result therefore demonstrates an outstanding illustration for the integration of the distinct properties of nanostructured carbon materials with different dimensionalities to achieve highly efficient devices. Together with the associated mechanism, it paves a valuable step for the further development of all carbon-based, cheap, and non-toxic optoelectronics devices with excellent performance.

$\mathrm{n}$ recent years, a family of carbon structures, such as fulleren $\mathrm{e}^{1,2}$, carbon nanodots ${ }^{3}$, carbon nanotubes $(\mathrm{CNTs})^{4-6}$, graphene ${ }^{7-10}$ and graphene quantum dots $(\mathrm{QDs})^{11-13}$, have attracted tremendous research interest and trial applications due to their superior and interesting chemical, physical, mechanical and electronic properties. Taking the advantage of abundant content in the earth, chemical stability, fine biocompatibility and non- toxicity, carbon based materials have emerged as attractive candidates for the development of bioimaging, medical diagnosis, catalysis, photovoltaic and many other optoelectronic devices ${ }^{14-16}$. Especially, the unique properties of nano-carbon based materials depend notably on material dimensionality, such as graphene QDs act as zero-dimensional materials, CNTs as one-dimensional (1D) materials, and graphene sheets as ideal twodimensional (2D) materials. Combining the unique properties arising from dimensionality and quantum confinement effects of nano-carbons, in both the pure forms of these materials and in hybrid structures, it should be able to generate unprecedented physical properties and provide a potential pathway for constructing all carbonbased devices with high perfromance ${ }^{17,18}$.

Among all the nano-carbon based materials, graphene QDs are emerging as one of the most attractive materials due to their size-tunable optical response, efficient multiple carrier generation, non-toxicity, superiority of a large optical absorptivity, and low cost ${ }^{19-21}$. In addition, graphene, an atomically thin film composed of a single layer of carbon, has also received intensive attention due to its outstanding electrical conductivity, high transparency and high robustness ${ }^{22-24}$. Graphene thus offers a promising conducting materials with low cost when compared with other frequently used conducting materials. Besides, it is also known that carbon-based conductive pastes are advantageous over other metal contacts for electrically contacting carbon nanostructures ${ }^{25-27}$. Therefore, stimulating by the superb optical and electrical properties of graphite QDs and 2D graphene, in this paper we provide the first attempt for the integration of zero-dimensional and $2 \mathrm{D}$ graphene to create novel all carbon-based photodetectors with ultrahigh sensitivity. Figure 1 shows the structure of all carbon-based photodetector fabricated on $\mathrm{Si} / \mathrm{SiO}_{2}$ substrate studied in the present work. Quite interestingly, it is found that the photocurrent responsivity can reach up to $4 \times 10^{7} \mathrm{AW}^{-1}$, which is comparable with the ultrahigh gain photodetectors reported previously ${ }^{28-32}$. Except the large absorption coefficient of graphite QDs and high conduction path provided by graphene, the remarkable ultrahigh responsivity can also be well understood by the spatial separation of photogenerated electron-hole pairs due to the appropriate band alignment across the interface between graphite QDs and graphene. Our result shown here therefore illustrates an excellent example for the creation of all carbon-based optoelectronic devices with high performance. It may open up a new route for the generation of many highly efficient devices with low cost, non-toxicity, and chemical stability. 


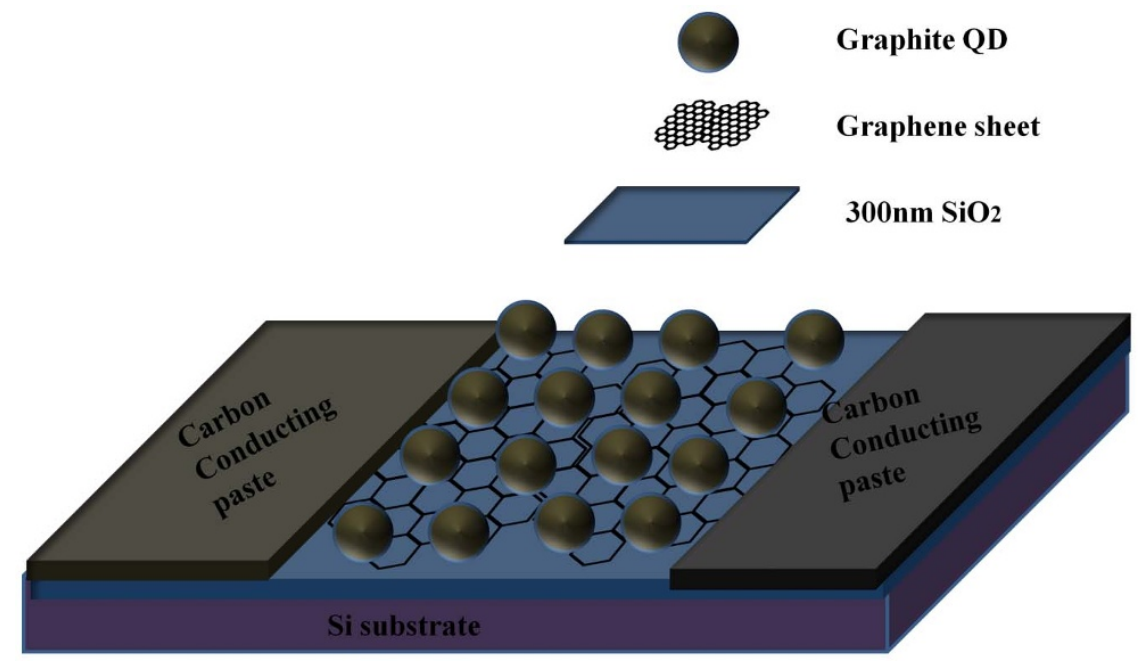

Figure 1 | Schematic of all carbon-based photodetector.

\section{Results}

The transmission electron microscopy (TEM) image of the synthesized graphite QDs on the surface of graphene film is shown in Fig. 2(a). It was found that graphite QDs have an average diameter of $6 \mathrm{~nm}$ and their distribution with a density of particle number of $2 * 10^{11} / \mathrm{cm}^{2}$ on the graphene device is relatively homo-dispersed. Raman spectrum (Fig. 2(b)) provided the convincing evidence for graphene fragment structure of the graphite QDs. The peak at $\sim 1588 \mathrm{~cm}^{-1}$ named $\mathrm{G}$ band corresponds to the $\mathrm{E}_{2 \mathrm{~g}}$ mode of graphite is related to the vibration of the $\mathrm{sp}^{2}$ - bonded $\mathrm{C}$ atoms in a $2 \mathrm{D}$ hexagonal lattice, while the peak at $1348 \mathrm{~cm}^{-1}$ named $\mathrm{D}$ band exhibits disorder due to the scattering at the edges. The relative intensity of $\mathrm{G} / \mathrm{D}$ band is $\sim 1.61$, similar to the previous report ${ }^{33}$. The quality and thickness of graphene sheet were also verified by Raman spectroscopy as shown in Fig. 2(c). A single symmetric $2 \mathrm{D}$ peak at $\sim 2690 \mathrm{~cm}^{-1}$ and a small G/2D ratio were observed, which shows the typical characteristics of single layer graphene with high quality. Figure 2(d) displays the photoluminescence spectrum of the graphite QDs at room temperature excited by a HeCd laser beam with a wavelength of $325 \mathrm{~nm}$, which shows that the emission peak wavelength is located at around $470 \mathrm{~nm}$. The UV-visible absorption spectrum of the graphite QDs in water measured by a PerkinElmer (Lambda 750) spectrometer shows a weak absorption band at around $360 \mathrm{~nm}$ arising from the band gap transition ${ }^{34}$. Figure 2(e) displays the X-ray diffraction (XRD) spectrum of the graphite QDs. The calculated lattice spacing is approximately $0.4 \mathrm{~nm}$, which is in good agreement with carbon based material with stratic disorder. The average particle size determined by Scherrer equation was $\sim 8 \mathrm{~nm}$, which is consistent with the observation of TEM image. It is widely known that the graphite QD is consisted by graphene like (sp2 like) species in $\mathrm{sp}^{3}$ (from oxygen- containing functional group) matrix $^{35-37}$. As shown in the previous reports ${ }^{38,39}$, the thickness of graphene oxide is approximately $0.7 \mathrm{~nm}$. To this regard, with the comparison of our TEM images, the graphite QD is composed of at least 10 to 15 layers of graphene sheets.

Figures 3(a) and (b) show the typical photocurrent and the responsivity measurements as a function of the source-drain bias under light illumination of a HeCd laser with different excitation intensities. Quite interestingly, when the graphite QDs were decorated on the graphene photodetector, the photocurrent drastically increases with increasing the illumination power. Notably, the high responsivity calculated under the optical power of $1.56 \mu \mathrm{W}$ and the external source-drain bias of $1 \mathrm{~V}$ is comparable with the magnitude of ultrahigh gain photodetectors in previous reports ${ }^{40-44}$. Here, the photoresponsivity was calculated according to the expression given by
$R=\frac{\text { Photocurrent }(A)}{\text { Incident Optical Power }(W)}=\frac{I_{p h}}{P_{0}}$. The small current change upon the illumination of graphene photodetector without the decoration of graphite QDs as shown in Fig. 3(a) indicates that the carrier accumulation in graphene sheet is magnified by the interaction with the charge transfer arising from the optically excited graphite QDs. Therefore, the high absorptivity of graphite QDs plays a crucial role in the behavior of the newly photosensitive detector.

To examine the underlying mechanism of the ultrahigh photosensitivity observed here, we have performed the dependence of photosensitivity on the excitation wavelength as shown in Fig. 4. The spectral responsivity of the device containing the graphite QDs basically follows the absorption spectrum of the graphite QDs as shown in Fig. 2(d). In addition, a weak photocurrent peak is also observed for the photon energy below the bandgap of graphite QDs. Markedly, the wavelength at around $470 \mathrm{~nm}$ of the weak photocurrent peak is consistent with the PL peak showing in Fig. 2(d), indicating that defects are responsible for both of the PL and photocurrent. A more detailed description of the photocurrent caused by defect states will be discussed below.

This result demonstrates that the spectral selectivity of the newly designed carbon-based photodetector can be manipulated well by the graphite QDs. The large photosensitivity of the graphite QDs/graphene photodetector therefore must involve the charge carrier transfer from graphite QDs to graphene and creates the spatial separation of photogenerated electrons and holes. Therefore, the excess photogenerated carriers can propagate along the excellent path provided by the graphene sheet, which leads to the giant enhancement of photocurrent.

In order to investigate the nature of the intrinsic charge transfer between graphene and graphite QDs, we have characterized the back-gated graphene field-effect transistor on $\mathrm{Si}$ wafer with a $300 \mathrm{~nm}$ thick $\mathrm{SiO}_{2}$ layer during the graphite QDs excitation as shown in Fig. 5(a). Before the deposition of graphite QDs, the characteristics $\mathrm{I}_{\mathrm{DS}}$ vs. $\mathrm{V}_{\mathrm{g}}$ of the graphene FET shown in Fig. 5(b) exhibits a Dirac point at $\mathrm{V}_{\mathrm{g}} \sim 1.6 \mathrm{~V}$, corresponding to a p type doping. The calculated field-effect mobilities are $\sim 1200 \mathrm{~cm}^{2} \mathrm{~V}^{-1} \mathrm{~s}^{-1}$ for the free carriers (electrons and holes), which are faster than those of many other bulk semiconductors.

After the decoration of graphite QDs, the I-V characteristics of the hybrid graphene transistor indicate an obvious positive shift $(\sim 48 \mathrm{~V})$ of the Dirac point due to the occurrence of hole accumulation in graphene film. Namely, a higher $V_{g}$ is needed to access to the Dirac point in the hybrid graphene transistor because the net negative charges in the graphite QDs can attract more photoinduced free 

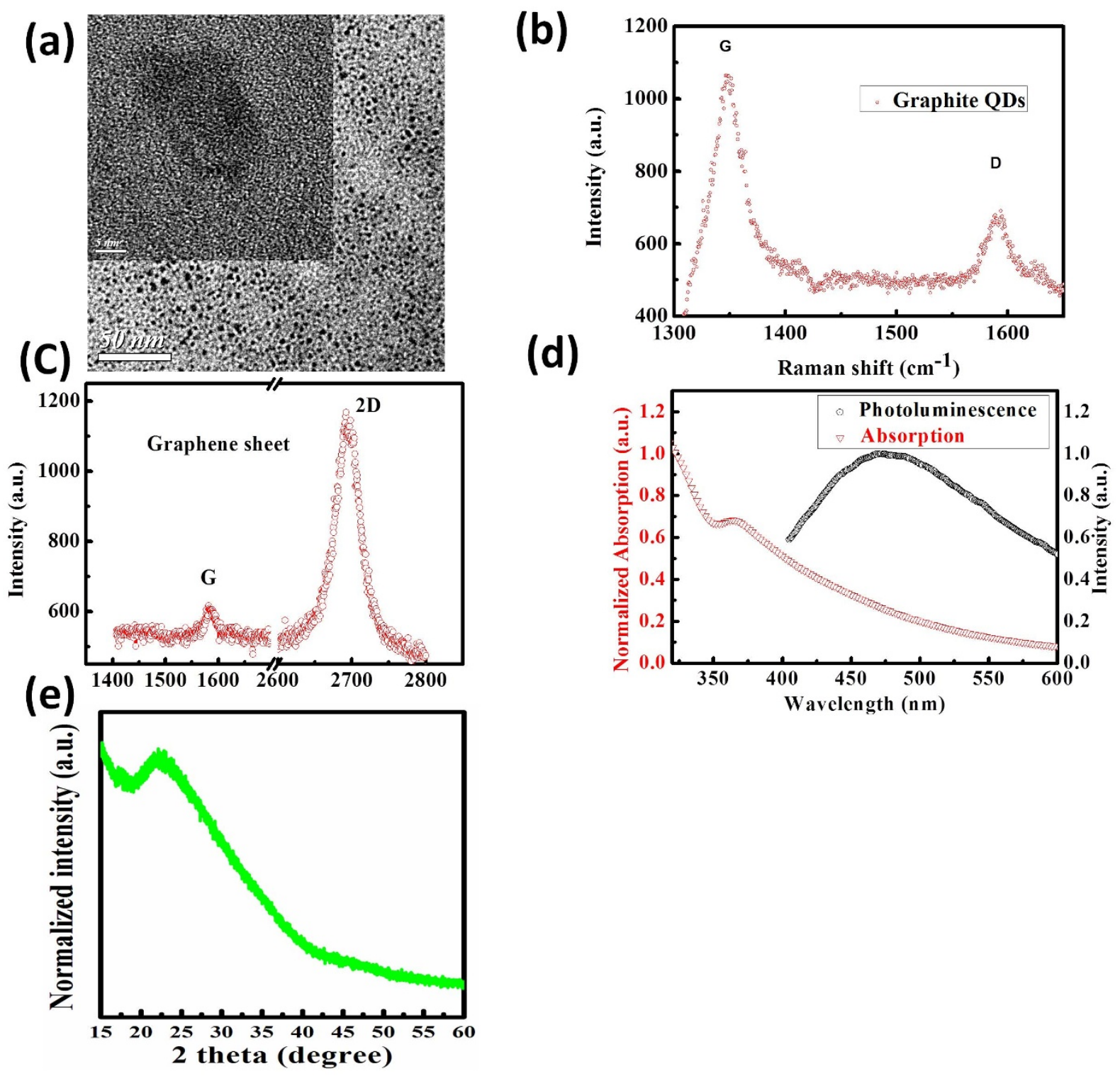

Figure $2 \mid$ (a) High resolution transmission electron microscope image of graphite quantum dots. (b) Raman spectra of graphite quantum dot. (c) Raman spectra of graphene sheet. (d) Photoluminescence and absorption spectra of graphite quantum dots. (e) X-ray diffraction spectrum (XRD) of graphite quantum dot.
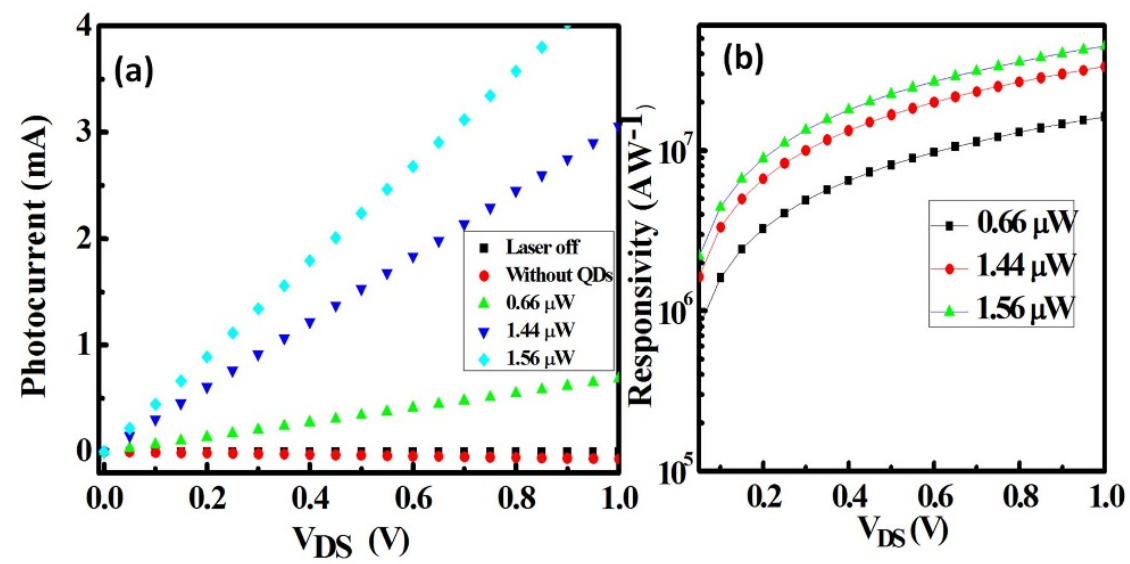

Figure $3 \mid$ (a) Photocurrent of all carbon-based photodetector for different optical powers as a function of drain-source voltage ( $\left.V_{D S}\right)$, showing a linear dependence on bias. The photocurrent for the pure graphene device without graphite quantum dots is also shown here, which indicates a negligible effect. (b) Responsivity of all carbon-based photodetector as functions of drain-source voltage characterized under different light irradiance. 


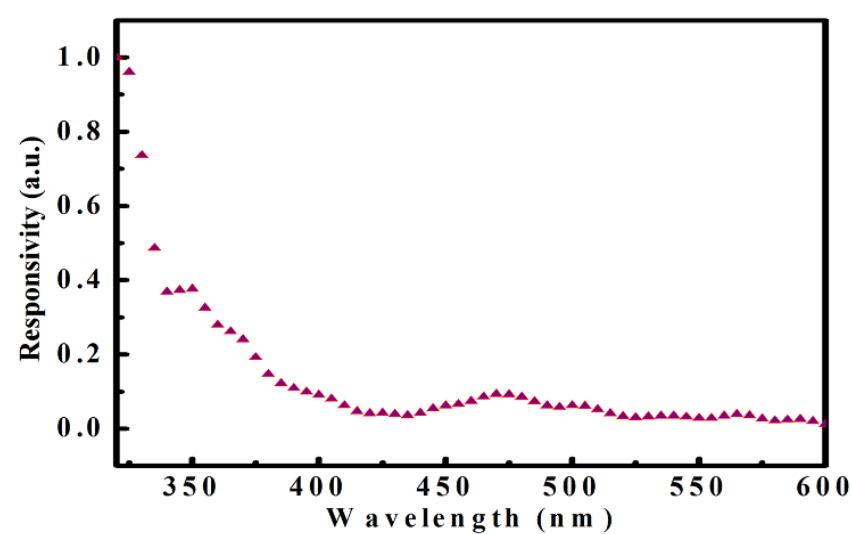

Figure 4 | Spectral responsivity of all carbon-based photodetector.

holes in the graphene film. Furthermore, the charge transfer creates an internal field and induces asymmetric transfer curves with the reduction of the electron mobility to $682 \mathrm{~cm}^{2} \mathrm{~V}^{-1} \mathrm{~s}^{-1}$ and a negligible effect on the hole mobility.

The theoretical prediction of the photocurrent gain can be estimated by the expression $G=\tau_{\text {lifetime }} \times Q E \times \mu \times \frac{V_{D S}}{A}$, where $\tau_{\text {lifetime }}$ is the photogenerated carrier lifetime, QE is quantum efficiency, $\mu$ is the carrier mobility, $V_{D S}$ is the applied bias between source and drain electrode, and $A$ is the area of the graphene sheet under light illumination. If we insert the experimental value of $\tau_{\text {lifetime }}=2 \mathrm{~s}, \mu=$
$1200 \mathrm{~cm}^{2} \mathrm{~V}^{-1} \mathrm{~s}^{-1}, V_{D S}=1 \mathrm{~V}, \mathrm{~A}=64 \mu \mathrm{m}^{2}$, and $\mathrm{QE}=26 \%$ estimated by the parallel plate capacitor model ${ }^{28}$, the theoretical gain has a value of about $1 \times 10^{9}$. The experimental gain number can be calculated by $G=\frac{\tau_{\text {lifetime }}}{\tau_{\text {transit }}}$, where $\tau_{\text {transit }}\left(\tau_{\text {transit }}=\frac{d^{2}}{\mu V_{D S}}\right)$. The obtained photocurrent gain is $3.75 \times 10^{9}$, which is in the same order of magnitude with the theoretical prediction.

\section{Discussion}

To understand the above behavior, let us consider the band alignment between the graphite QDs and two dimensional graphene film as shown in Fig. 6. According to the absorption spectrum shown in Fig. 2(b), the graphite QDs have a band gap of about $3.441 \mathrm{eV}$. Having symmetric linear energy dispersion relations in both valence and conduction bands near the band edges in graphite QDs make equal effective masses for both electrons and holes. According to the previous report ${ }^{41}$, the work functions of the highest occupied states and the lowest unoccupied states of the graphite QDs are estimated to be $6.14 \mathrm{eV}$ and $2.7 \mathrm{eV}$, respectively, as shown in Fig. 5. Because graphite QDs are found to be of p-type nature, the corresponding Fermi energy is close to its highest occupied states. The energy level of the Dirac point of intrinsic graphene film is at about $4.5 \mathrm{eV}$. Therefore, according the band alignment as shown in Fig. 6(a), the photogenerated holes in graphite QDs will transfer to the graphene sheet, cause the reduction of the hole carriers in graphite QDs and induce a p-type doping effect in graphene, which leads to the shift of the Dirac point from a lower voltage to a higher voltage. Such carrier transfer has been known to allow a more effective charge separation in graphite QDs and consequently inhibit the electron-hole recombination ${ }^{32,41,42}$. (a)

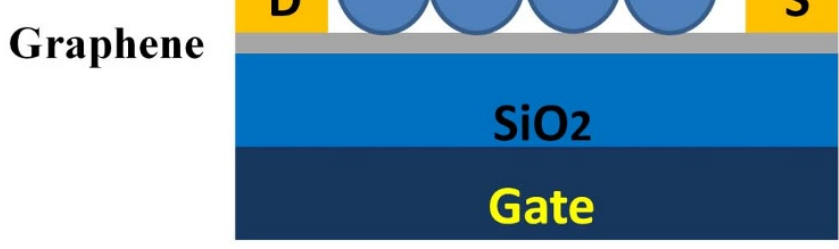

(c)

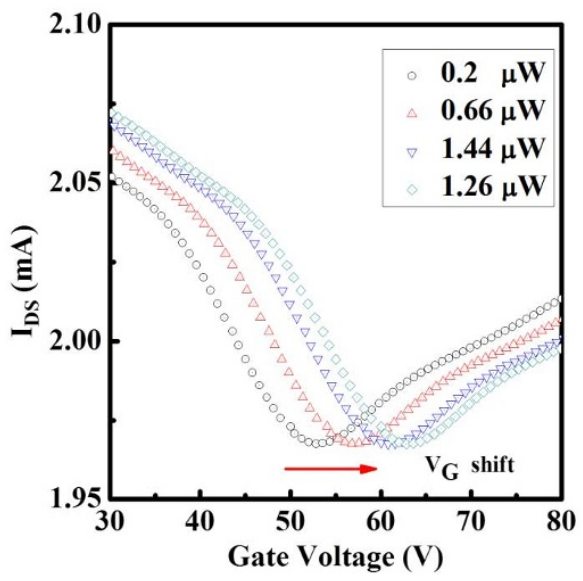

(b)

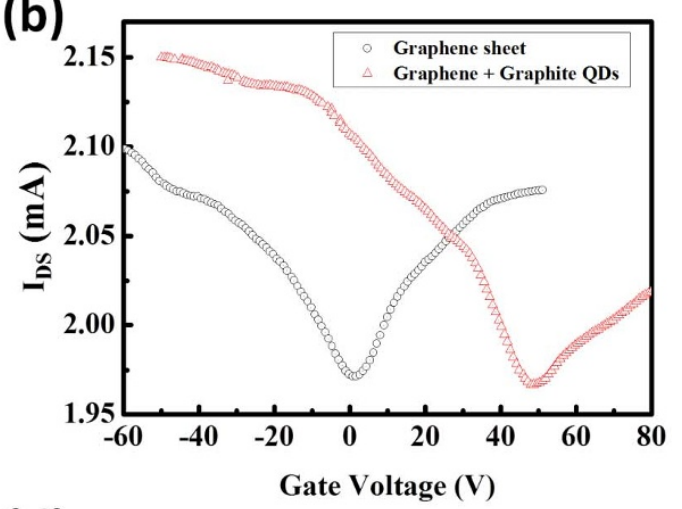

(d)

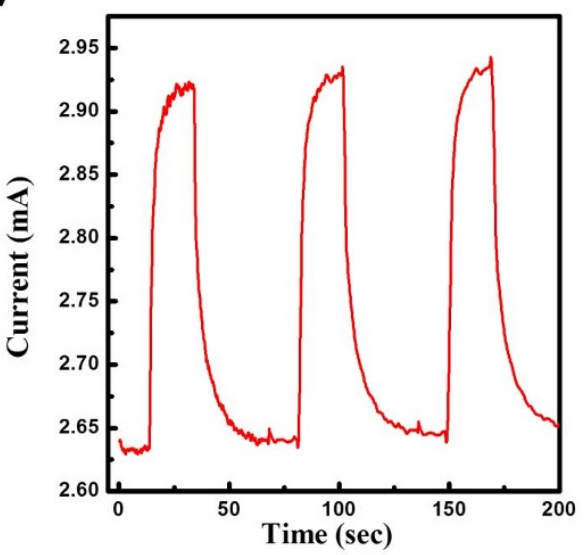

Figure $5 \mid$ (a) Schematic of graphite quantum dots/graphene phototransistor. (b) Transfer characteristics of graphene transistors with and without the addition of graphite QDs on the graphene sheet. (c) Transfer characteristics of graphite quantum dots/graphene phototransistor characterized under UV light irradiation with different power (d) Photocurrent response switching measurements for graphite quantum dots/graphene phototransistor under UV light irradiation. 
(a)

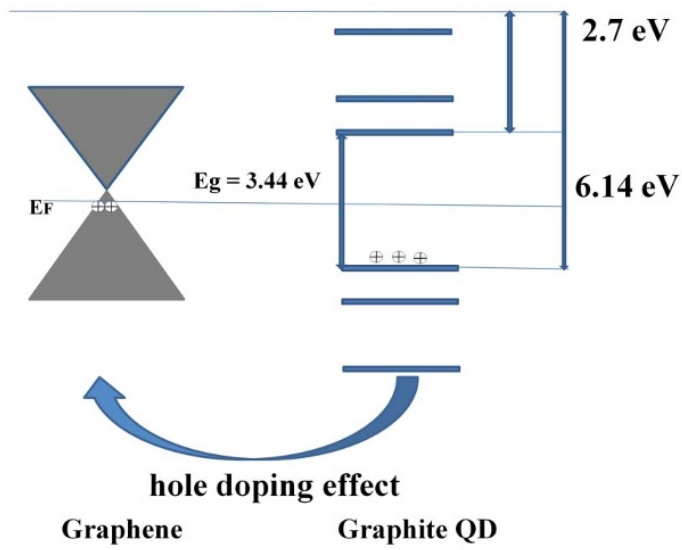

(b)

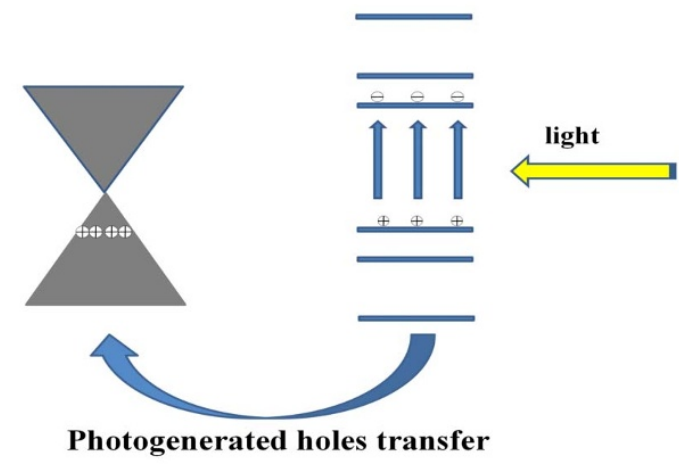

Figure $6 \mid$ (a) Schematic band diagram of graphite quantum dot-graphene hybrid device before light illumination. $\mathrm{E}_{\mathrm{g}}$ corresponds to the bandgap of graphite quantum dots. (b) Under light illumination, hole can transfer from graphite quantum dots to graphene sheet. The photogenerated electrons are trapped in graphite quantum dots.

We now examine the influence of optical excitation. Under UV light illumination, the Dirac point of the transfer characteristics of the hybrid graphene phototransistor shifts toward a higher gate voltage with increasing irradiation power as shown in Fig. 5(c). This behavior can be understood based on the band alignment between graphite QDs and graphene. According to the band alignment as shown in Fig. 6, the photogenerated holes in graphite QDs will transfer into graphene sheet and contribute to the photocurrent. Therefore, both of the giant photocurrent gain and the shift of the Dirac point toward a large gate voltage can be well interpreted. Besides, it is worth noting that the turn-on transient shown in
Fig. 4(d) is characterized by a relatively fast increase in the photocurrent, and followed by a relatively slow decay to a steady state value. This behavior can also be understood in terms of the charge transfer as shown in Fig. 6. The fast increase in the photocurrent under light illumination arises from the fast carrier transfer process from graphite QD to graphene and the high carrier mobility of graphene sheet. And, the slow decay after turning off the light is due to the spatial separation of electrons and holes, which defers the electron- hole recombination.

The above mechanism can also be used to interpret the photocurrent induced by the transition of defect states. We can clearly see that the photocurrent peaks at around $360 \mathrm{~nm}$ and $470 \mathrm{~nm}$ in Fig. 4 are consistent with the absorption and PL spectra as shown in Figs. 2(b) and (d). According to previous reports ${ }^{35-37,45,46}$, the graphene quantum dots consist of numerous disorder induced defect states within the $\pi-\pi^{*}$ gap and exhibit a broad prominent PL spectrum. Upon light illumination, the electrons in the valence band of QDs can be excited to the defect states and create holes in the valence band. The photogenerated holes due to the existence of defect absorption can transfer into graphene sheet and contribute to the detected photocurrent.

To further confirm the charge transfer of photogenerated holes from graphite QDs to graphene, we have performed PL measurement as shown in Fig. 7(a). It indicates that the PL intensity of graphite QD is quenched in the presence of graphene sheet. The PL quenching in the graphite QD and graphene composite is consistent with the expectation that photogenerated holes can transfer from graphite QD to graphene, which enables to reduce the radiative recombination probability in the graphite QD. In addition, Fig. 7(b) shows the time resolved photoluminescence (TRPL) spectra measured by a near infrared ultrafast laser (Uranus 005, Polar Onyx, CA) with a wavelength $1040 \mathrm{~nm}$ as the source of three photon excitation. It exhibits that after the decoration of graphite QDs on graphene, the PL lifetime decreases from 0.133 ns to 0.09 ns. This implies that due to the charge transfer process, there exists an additional channel to reduce the recombination probability, and leads to the reduction of the photogenerated carrier lifetime. Therefore, both PL measurements provide an additional evidence to support our proposed mechanism for the interpretation of the ultrahigh gain photodetector made with graphite QDs and graphene.

Finally, let us compare the performance of the photodetectors consisted of graphite QDs and graphene sheet developed here with simple graphene photodetectors. It is clear that the photocurrent gain in our new device is much higher due to the large absorption coefficient of graphite QDs and the charge transfer caused by the band alignment between graphite QDs and graphene. However, the trade-off is that the response speed becomes slow because of the additional process for the spatial separation of photogenerated
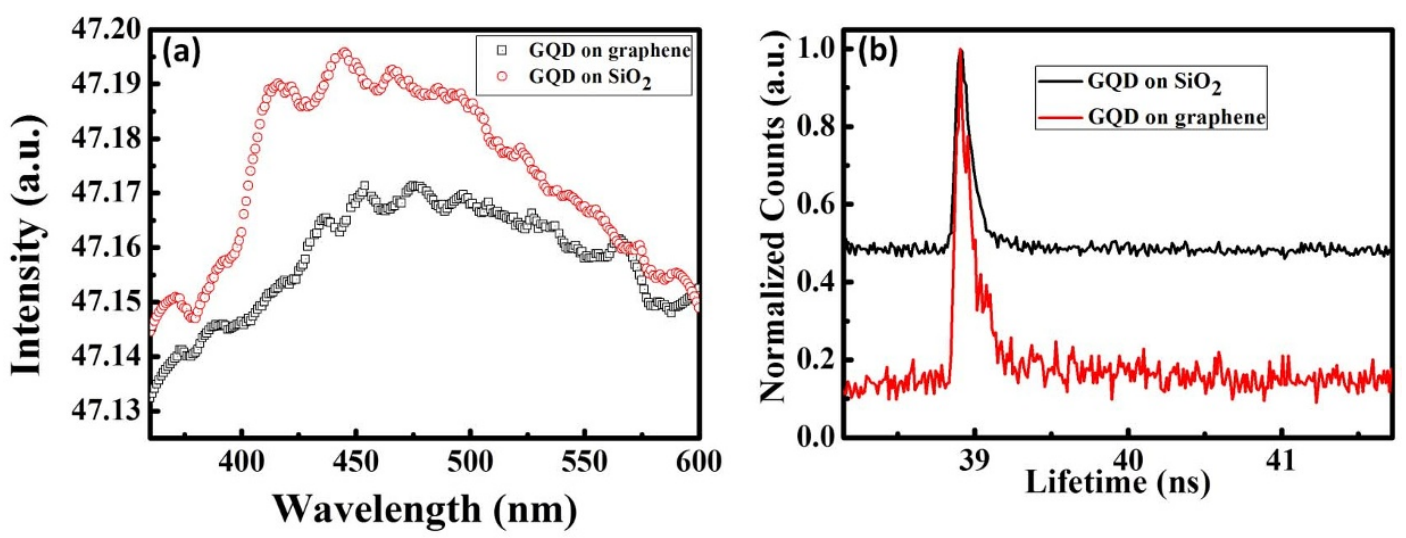

Figure $7 \mid$ (a) Photoluminescence spectra of graphite quantum dots on graphene sheet and $\mathrm{SiO}_{2}$ substrate. (b) Time-resolved photoluminescence decay spectra for graphite quantum dots on graphene sheet and $\mathrm{SiO}_{2}$ substrate. 
electrons and holes, and the operational wavelength range for the high responsivity is restricted to the band gap absorption of quantum dots. These features arise from the inherent nature of the photodetectors with similar structure as shown in previous reports $\mathrm{s}^{28,42-45}$.

In summary, we have demonstrated that the ultrahigh sensitivity of photoresponse can be obtained based on the integration of zero dimensional graphite QDs and 2D graphene sheet. The underlying mechanisms arise from several important factors, including the large absorptivity of graphite QDs and high conductivity of graphene sheet. The charge transfer due to the appropriate band alignment between graphite QDs and graphene also plays a decisive role, which creates the spatial separation of photogenerated electrons and holes, prolongs the photoinduced electron lifetime, and improves the photocurrent responsivity. It is stressed here that the drastic improvement of photocurrent gain of the hybrid photodetectors made with of all carbon-based materials shown here can serve as an excellent illustration for the integration of the unique properties of nano - carbons with different dimensionalities. It can open up a new route for the creation of all carbon-based devices with low cost, nontoxicity, and high performance.

\section{Methods}

For the synthesis of QDs, $2 \mathrm{~g}$ 11-aminoundecanoic acid was first dissolved in $25 \mathrm{ml}$ DI water then neutralized by $1 \mathrm{M}$ sodium hydroxide aqueous solution. Afterward, $2 \mathrm{~g}$ citric acid was added to the above solution. With vigorous stirring, a thick precipitation was observed within a few minutes. The precipitation was collected by filtration and allowed to dry at $85^{\circ} \mathrm{C}$ for one day. The dried precipitant was then grounded into fine powder and directly oxidized in air at $300^{\circ} \mathrm{C}$ for 2 hours in muffle oven under ambient atmosphere. The QD was extracted by hot water, solid impurity was removed by centrifuge. For purification, $\mathrm{pH}$ of extracted QD was adjusted to 2 by concentrated hydrochloric acid, which yields bulk precipitation. The soluble impurity was then removed by centrifuge. The precipitant was then treated with $50 \mathrm{ml} 0.1 \mathrm{M}$ $\mathrm{NaOH}$, which causes the dissolution of precipitant. Minor insoluble impurity was then removed by centrifuge. The purification procedure was repeated for 3 times. Finally, a clear and deep-brown dense colloidal quantum dots solution with long period stability was obtained. According to previous reports ${ }^{47,48}$, the deep brown color may arise from the aggregation of the QDs. The precursor for quantum dot was mainly from protonation between 11 -aminoundecanoic acid and citric acid. The lateral thermal treatment incorporated the carbon in precursor to graphite QDs, meanwhile, the amine linkage from amine-carboxylic group condensation was formed. With the above regards, particle size control could be potentially facilitated by the manipulation of the carbon source in precursor ${ }^{49}$.

High quality graphene sheet was synthesized on copper foils by using efficient chemical vapor deposition (CVD) method and transferred with the aid of polymethylmethacrylate (PMMA) following the method reported by Li et $\mathrm{a}^{33,50}$. This method allows to produce a large scale production of graphene-based devices with high yield. The hybrid graphite QDs-graphene photodetector was fabricated on a $\mathrm{Si} /$ $\mathrm{SiO}_{2}$ substrate, where the thickness of $\mathrm{SiO}_{2}$ layer is approximately $300 \mathrm{~nm}$. After transferring the CVD graphene on the $\mathrm{SiO}_{2}$ surface, carbon conductive paste was adhered on both sides of graphene film as source and drain electrodes. The channel lengths, defined as the distance between source and drain was $8 \mu \mathrm{m}$ and the channel width (W) was maintained at $0.1 \mathrm{~mm}$. Graphite QDs were then spin-casted onto the graphene film by instilling the graphite QD drops into the surface. The optimized spin speed was set to $1000 \mathrm{rpm}$ throughout the film preparation, and then moved into a glove-box filled with high purity $\mathrm{N}_{2}$. In order to improve the interface contact between graphite $\mathrm{QDs}$ and graphene, the device was baked at $100^{\circ} \mathrm{C}$ for two minutes to evaporate the deposited chemical solvent before further measurements. The Raman scattering spectra used to examine the quality of graphite QDs and graphene were measured by a Jobin Yvon T64000 system. For the photocurrent measurement, we use a He-Cd laser working at $325 \mathrm{~nm}$ or a xenon- arc lamp dispersed by monochoromator as the excitation light sources. A measurement system of Keithley 236 was utilized to supply the DC voltage and to record the photocurrent.

1. Wang, Y. Photoconductivity of fullerene-doped polymers. Nature 356 585-587 (1992)

2. Tenne, R. Inorganic nanotubes and fullerene-like nanoparticles. Nat. Nanotech. 1, 103-111 (2006).

3. Baker, S. N. \& Baker, G. A. Luminescent carbon nanodots: emergent nanolights. Angew. Chem. Int. Ed. 49, 6726-6744 (2010).

4. Tans, S. J., Verschueren, A. R. M. \& Dekker, C. Room-temperature transistor based on a single carbon nanotube. Nature 393, 49-52 (1998).

5. Bachtold, A., Hadley, P., Nakanishi, T. \& Dekker, C. Logic circuits with carbon nanotube transistors. Science 294, 1317-1320 (2001).

6. Baughman, R. H. et al. Carbon nanotube actuators. Science 284, 1340-1344 (1999).
7. Nair, R. R. et al. Fine structure constant defines visual transparency of graphene. Science 320, 1308 (2008).

8. Geim, A. K. Graphene: status and prospects. Science 324, 1530-1534 (2009).

9. Geim, A. K. \& Novoselov, K. S. The rise of graphene. Nat. Mater. 6, 183-191 (2007).

10. Chen, Z. et al. Three-dimensional flexible and conductive interconnected graphene networks grown by chemical vapour deposition. Nat. Mater. 10, 424-428 (2001).

11. Ponomarenko, L. A. et al. Chaotic Dirac billiard in graphene quantum dots. Science 320, 356-358 (2008).

12. Dirks, T. et al. Transport through Andreev bound states in a graphene quantum dot. Nat. Phys. 7, 386-390 (2011).

13. Yan, X., Cui, X., Li, B. \& Li, L. S. Large, solution-processable graphene quantum dots as light absorbers for photovoltaics. Nano Lett. 10, 1869-1873 (2010).

14. Huynh, W. U., Dittmer, J. J. \& Alivisatos, A. P. Hybrid nanorod-polymer solar cells. Science 295, 2425-2427 (2002).

15. Jaiswal, J. K., Goldman, E. R., Mattoussi, H. \& Simon, S. M. Use of quantum dots for live cell imaging. Nat. Methods 1, 73-78 (2004).

16. Castro Neto, A. H. et al. The electronic properties of graphene. Rev. Mod. Phys. 81, 109-162 (2009).

17. Ramuz, M. P. et al. Evaluation of Solution-Processable Carbon-Based Electrodes for All-Carbon Solar Cells. ACS Nano. 6, 10384-10395 (2012).

18. Qi, J. S. et al. The Possibility of Chemically Inert, Graphene-Based All-Carbon Electronic Devices with 0.8 eV Gap. ACS Nano. 5, 3472-3485 (2011).

19. Zhu, S. et al. Graphene quantum dots with controllable surface oxidation, tunable fluorescence and up-conversion emission. RSC Adv. 2, 2717-2720 (2012).

20. Li, Y. et al. An Electrochemical Avenue to Green-Luminescent Graphene Quantum Dots as Potential Electron-Acceptors for Photovoltaics. Adv. Mater. 23, 776-780 (2011)

21. Gupta, V. et al. Luminscent graphene quantum dots for organic photovoltaic devices. J. Am. Chem. Soc. 133, 9960-9963 (2011).

22. Novoselov, K. S. et al. Two-Dimensional Gas of Massless Dirac Fermions in Graphene. Nature 438, 197-200 (2005).

23. Yu, Y. J. et al. Tuning the Graphene Work Function by Electric Field Effect. Nano Letters 9, 3340-3434 (2009).

24. Stankovich, S. et al. Graphene-Based Composite Materials. Nature 442, 282-286 (2006).

25. Tung, V. C. et al. Low-temperature solution processing of graphene- carbon nanotube hybrid materials for high-performance transparent conductors. Nano letter. 9, 1949-1955 (2009).

26. King, P. J., Khan, U., Lotya, M., De, S. \& Coleman, J. N. Improvement of transparent conducting nanotube films by addition of small quantities of graphene. ACS Nano. 4, 4238-4246 (2010).

27. Burghard, M., Klauk, H. \& Kern, K. Carbon-based field-effect transistors for nanoelectronics. Adv. Mater. 21, 2586-2600 (2009).

28. Konstantatos, G. et al. Hybrid graphene-quantum dot phototransistors with ultrahigh gain. Nat. Nanotechnol. 7, 363-368 (2012).

29. Lee, E. J. H. et al. Contact and edge effects in graphene devices. Nat. Nanotechnol. 3, 486-490 (2008).

30. Park, J., Ahn, Y. H. \& Ruiz-Vargas, C. Imaging of photocurrent generation and collection in single-layer graphene. Nano Lett. 9, 1742-1746 (2009).

31. Xia, F., Mueller, T., Lin, Y.-M., Valdes-Garcia, A. \& Avouris, P. Ultrafast graphene photodetector. Nature Nanotech. 4, 839-843 (2009).

32. Sun, Z. H. et al. Infrared Photodetectors Based on CVD-Grown Graphene and PbS Quantum Dots with Ultrahigh Responsivity. Adv. Mater. 24, 5878-5883 (2012).

33. Li, Y. et al. An Electrochemical Avenue to Green-Luminescent Graphene Quantum Dots as Potential Electron-Acceptors for Photovoltaics. Adv. Mater. 23, 776-780 (2011).

34. Reina, A. et al. Large area, few-layer graphene films on arbitrary substrates by chemical vapor deposition. Nano Lett. 9, 30-35 (2009).

35. Kian, P. L., Qiaoliang, B., Goki, E. \& Manish, C. Graphene oxide as a chemically tunable platform for optical applications. Nat. Chem. 2, 1015-1024 (2010).

36. Galande, C. et al. Quasi-molecular fluorescence from graphene oxide. Sci. Rep. 1, 85 (2011).

37. Jingzhi, S. et al. The Origin of Fluorescence from Graphene Oxide. Sci. Rep. 2, 972 (2012).

38. Goki, E., Giovanni, F. \& Manish, C. Large-area ultrathin films of reduced graphene oxide as a transparent and flexible electronic material. Nat. Nanotech. 3, 270-274 (2008).

39. Dikin, D. A. et al. Preparation and characterization of graphene oxide paper. Nature 448, 457-460 (2007).

40. Sargent, E. H. Photodetectors: A sensitive pair. Nat. Nanotech. 7, 349-350 (2012).

41. Konstantatos, G. \& Sargent, E. H. PbS colloidal quantum dot photoconductive photodetectors: Transport, traps, and gain. Appl. Phys. Lett. 91, 173505-173508 (2007).

42. Zhang, D., Gan, L., Cao, Y., Wang, Q. \& Guo, X. Understanding Charge Transfer at PbS-Decorated Graphene Surfaces toward a Tunable Photosensor. Adv Mater. 24, 2715-2720 (2012).

43. Konstantatos, G. \& Sargent, E. H. Nanostructured materials for photon detection Nat. Nanotech. 5, 391-400 (2010).

44. Shen, J., Zhu, Y., Chen, C., Yang, X. \& Li, C. Facile preparation and upconversion luminescence of graphene quantum dots. Chem. Commun. 47, 2580-2582 (2011). 
45. Eda, G. et al. Blue photoluminescence from chemically derived graphene oxide. Adv. Mater. 22, 505-509 (2009).

46. Sun, Y. P. et al. Quantum-sized carbon dots for bright and colorful photoluminescence. J. Am. Chem. Soc. 128, 7756-7757 (2006).

47. Tang, L. et al. Deep ultraviolet photoluminescence of water-soluble self passivated graphene quantum dots. ACS Nano 6, 5102-5110 (2012).

48. Li, Y. H. et al. Fluorescent graphene quantum dots with a boronic acid appended bipyridinium salt to sense monosaccharides in aqueous solution. Chem. Commun 49, 5180-5182 (2013).

49. Dikin, D. A. et al. Preparation and characterization of graphene oxide paper. Nature 448, 457-460 (2007).

50. Li, X. et al. Large-area synthesis of high-quality and uniform graphene films on copper foils. Science 324, 1312-1314 (2009).

\section{Acknowledgements}

This work was supported by the National Science Council and Ministry of Education of the Republic of China.

\section{Author contributions}

Y.F.C. planned and supervised the project; M.L.L. advised on the project; S.H.C. and T.M.W. designed and performed experiments; S.H.C., W.C.T. and T.M.W. made samples and the structural measurements; S.H.C., J.Y.C. and T.M.W. performed the electrical measurements; and Y.F.C. and S.H.C. analyzed data and wrote the manuscript. All authors discussed and commented on the manuscript.

\section{Additional information}

Competing financial interests: The authors declare no competing financial interests.

How to cite this article: Cheng, S. et al. All Carbon-Based Photodetectors: An eminent integration of graphite quantum dots and two dimensional graphene. Sci. Rep. 3, 2694; DOI:10.1038/srep02694 (2013).

By nc No NonCommercial-NoDerivs 3.0 Unported license. To view a copy of this license, visit http://creativecommons.org/licenses/by-nc-nd/3.0 